\title{
TERAPEUTAS OCUPACIONAIS E OS CENTROS DE CONVIVÊNCIA E COOPERATIVAS: NOVAS AÇÕES DE SAÚDE*
}

\section{OCCUPATIONAL THERAPISTS AND 'CENTROS DE CONVIVÊNCIA E COOPERATIVAS': NEW HEALTH ACTIONS}

\author{
Roseli Esquerdo Lopes ${ }^{(1)}$, Adriana Leão ${ }^{(2)}$
}

\begin{abstract}
LOPES, R. E.; LEÃO, A. Terapeutas ocupacionais e os centros de convivência e cooperativas: novas ações de saúde. Rev. Ter. Ocup. Univ. São Paulo, v. 13, n. 2, p. 56-63, maio/ago. 2002.

RESUMO: O objetivo desta pesquisa foi conhecer a atuação dos terapeutas ocupacionais nos Centros de Convivência e Cooperativas (CeCCos), desde sua implantação na cidade de São Paulo (1989) até 2000, dentro do contexto mais amplo no qual se inserem, ou seja, dos serviços municipais de saúde. Nesse período, que compreende três gestões municipais (89/92, 93/96 e 97/2000), ocorreram profundas e significativas alterações no campo da assistência pública à saúde na cidade de São Paulo. Analisou-se através de bibliografia, documentos, e entrevistas, as gestões municipais do período 1989-2000, no que se refere às suas proposições para a área da saúde, articulando-as com as ações desenvolvidas pelos CeCCos. Verificando-se, também, em que medida as modificações políticas e sociais influenciaram na proposta desse serviço. Buscouse: conhecer a atuação dos terapeutas ocupacionais junto às equipes multidisciplinares e à população usuária dos CeCCos; discutir a contribuição específica deste profissional no processo de implementação desse serviço; compreender de que forma o terapeuta ocupacional utiliza o recurso "atividades", a partir de sua ótica e também da ótica dos demais profissionais; e compreender o uso do recurso "atividades" nos CeCCos que não contam em sua equipe com o terapeuta ocupacional. Constatou-se que os CeCCos lograram constituir-se em importantes inovações de intervenção na área social com interfaces no campo da saúde, cultura e educação. O terapeuta ocupacional teve papel relevante na implantação e manutenção desse equipamento e o recurso "atividades" representou um eixo importante para o acolhimento e para a inclusão social dos usuários dos CeCCos.
\end{abstract}

DESCRITORES: Terapia ocupacional/recursos humanos. Comportamento cooperativo. Prestação de cuidados de saúde. Equipe de assistência ao paciente/tendências. Política de saúde/ tendências.

\footnotetext{
* Este artigo é resultado da pesquisa Terapeutas Ocupacionais e Centros de Convivência e Cooperativas: novos técnicos e novas ações de saúde na cidade de São Paulo, que contou com o apoio do CNPq. Parte dessa mesma pesquisa foi apresentada no VII Congresso Brasileiro de Terapia Ocupacional, Porto Alegre, out. 2001.

(1) Professora Adjunta do Departamento de Terapia Ocupacional da Universidade Federal de São Carlos - Doutora em Educação pela Universidade Estadual de Campinas.

(2) Terapeuta Ocupacional formada pela Universidade Federal de São Carlos.

Endereço para correspondência: Departamento de Terapia Ocupacional da Universidade Federal de São Carlos. Rodovia Washington Luiz, Km 235 - Caixa Postal, 676. CEP: 13565-905 - São Carlos, SP. e.mail: relopes@ power.ufscar.br e drileão2002@yahoo.com.br
} 


\section{INTRODUÇÃO}

$\mathrm{N}$ a cidade de São Paulo, em 1989, foi eleito para a Prefeitura do Município um governo preocupado em propor políticas específicas com o objetivo da adoção efetiva do Sistema Único de Saúde (SUS), a partir dos municípios (LOPES, 1999). Neste sentido, este governo, representado pela Prefeita Luiza Erundina, passou a desenvolver ações de saúde direcionadas a grupos populacionais até então excluídos dos serviços municipais, buscando garantir a assistência à saúde $\boldsymbol{a}$ todos os cidadãos, como preconiza o SUS, criado a partir da Constituição de 1988 (BRASIL, 1988).

Dessa forma, novos equipamentos e novas ações de saúde foram implementados, incluindo na assistência pública municipal a população portadora de transtorno mental e a população portadora de deficiência.

Para a efetivação dessas ações, além dos CeCCos, foram criados Hospitais-Dia, Enfermarias e Emergências Psiquiátricas em Hospitais Gerais, equipes de saúde mental e equipes de reabilitação nas Unidades Básicas de Saúde e Unidades de Reabilitação. Houve a ampliação do número de vagas existentes para profissionais que atendessem a essa demanda populacional, dentre eles, o terapeuta ocupacional, além de fisioterapeuta, fonoaudiólogo, psicólogo, psiquiatra, assistente social e enfermeiro (BARROS et al., 1995).

Assim, o terapeuta ocupacional é incorporado ao serviço público municipal, através de dois concursos realizados pela Prefeitura, que ampliaram o número de vagas desta categoria de três para 241. Esses técnicos vieram a desenvolver ações de saúde nos serviços gerais, assim como participaram das equipes multidisciplinares dos serviços direcionados àqueles grupos populacionais (BARROS et al., 1995).

Surgem neste contexto os Centros de Convivência e Cooperativas (CeCCos), com o objetivo de trabalhar especificamente a questão da inserção e da integração de grupos freqüentemente discriminados (pessoas portadoras de transtorno mental, pessoas portadoras de deficiência, portadores de HIV, crianças e adolescentes em situação de risco e idosos).

Esse equipamento ao ser implantado em espaços públicos (parques, centros esportivos, centros comunitários) propiciaria o encontro da população dita 'normal' com aquela população alvo. A convivência entre essas populações se efetivaria através do uso de oficinas, mediadas por uma equipe multidisciplinar de técnicos das áreas da saúde, da educação e da cultura. $\mathrm{O}$ CeCCo funcionaria como retaguarda dos serviços de saúde em geral e fundamentalmente dos serviços de saúde mental.
O projeto dos Centros de Convivência e Cooperativas foi iniciado experimentalmente em 1989, e foi oficializado no início de 1990, por portaria intersecretarial entre as Secretarias Municipais de Saúde (SMS), de Serviços e Obras, através de seu Departamento de Parques e Áreas Verdes (DEPAVE), e de Educação. Até o final de 1992, 18 CeCCos estavam em funcionamento na cidade de São Paulo (LOPES, 1999).

A partir de 1993, com a nova gestão municipal, desencadearam-se mudanças na política municipal de saúde, que provocaram, entre outras coisas, um importante arrocho salarial e o sucateamento dos serviços de saúde, com a inversão da curva de investimento municipal em saúde, ascendente até 1993 e que passou então a decrescer acentuadamente, para só ser retomada em 1995 (COHN; ELIAS, 1997) com a proposta do PAS (Plano de Atendimento à Saúde). Surge uma nova configuração para a atenção em saúde em geral, assim como para a saúde mental e saúde da pessoa portadora de deficiência (LOPES, 1999).

Com a implantação do PAS, em 1996, houve a desativação de alguns serviços de saúde que faziam parte do Programa de Saúde Mental e, dentre eles, três dos dezoito CeCCos existentes. Além disso, houve um êxodo dos profissionais que não aderiram ao sistema $\mathrm{e}$ permaneceram no quadro da Prefeitura: alguns poucos permaneceram em serviços da SMS que não foram absorvidos pelo PAS; outros, a maioria, foram transferidos para diversas secretarias; outros ainda, foram para serviços da rede pública estadual e mesmo para a rede privada, saindo da Prefeitura $(\mathrm{COHN}$; ELIAS, 1997; LOPES, 1999).

A gestão 97/2000 deu continuidade às propostas da gestão que a antecedeu. Entretanto, a maior parte dos 15 CeCCos se manteve.

Analisamos neste trabalho as propostas dos Centros de Convivência e Cooperativas, no período 1989-2000. Enfocaremos especialmente o trabalho desenvolvido pelos terapeutas ocupacionais junto às equipes multidisciplinares e à população alvo, buscando conhecer, apresentar e avaliar sua contribuição específica no processo de implantação e manutenção desse equipamento inovador e singular.

\section{METODOLOGIA}

Para apreender de que forma constituíram-se os novos serviços de saúde criados, especificamente o Centro de Convivência e Cooperativas e a análise sobre a atuação dos novos técnicos, sobretudo dos terapeutas 
ocupacionais, e, para além disso, saber qual o grau de permanência dessas inovações após a gestão que a implantou, abrangendo o período 1989-2000, optou-se pela utilização da metodologia de análise proposta por Offe e Lenhardt (1984). Buscou-se então: compreender como se configuraram as inovações sócio-políticas nas políticas sociais da gestão municipal; demonstrar o motivo pelo qual foram consideradas 'inovações' a implantação de novos serviços de saúde pelo governo municipal de São Paulo em 89/92; analisar as prioridades que foram dadas para a área da saúde pelas gestões do período sob análise (89/92, 93/96 e 97/2000), comparando-se também as modificações ocorridas com a proposta dos $\mathrm{CeCCos}$.

Ainda, a fim de compreender de que forma o terapeuta ocupacional utiliza os recursos "atividade" e "oficinas", a partir da sua ótica e também da ótica dos demais profissionais que compõem a equipe multidisciplinar do serviço, e como se dá a utilização do recurso "atividade" nos CeCCos que não contam com o terapeuta ocupacional, adotamos, como procedimentos de pesquisa, além do estudo bibliográfico e documental, entrevistas semiestruturadas com os profissionais que compõem (ou compuseram) as equipes, visitas a todos os $\mathrm{CeCCos}$ da cidade em funcionamento, no ano de 2000, e a observação participativa em dois deles.

\section{Os Centros de Convivência e Cooperativas}

\section{A proposta}

Diante de uma nova proposição de assistência à saúde que se configurava na cidade de São Paulo, a gestão 89/92 com o intuito de promover a universalidade e a eqüidade na atenção a grupos populacionais discriminados e sem acesso aos vários serviços municipais, não só de saúde, mas também de educação, cultura e lazer, buscava ações que confrontassem a discriminação e a segregação presentes na cultura baseada em idéias manicomiais e excludentes (LOPES, 1999).

Nesse sentido o CeCCo seria o equipamento adequado para este fim, pois exerceria o papel de:

"colaborador diferenciado no processo terapêutico dos que sofrem psiquicamente e dos que sofrem pela condição de estigmatizado. A terapêutica, neste contexto, se inscreve sob outro contrato, que se distancia do usualmente padronizado, tanto pela formação acadêmica quanto pela prática profissional privada e sua fiel reprodução nos espaços de saúde pública. (...) Sua vocação é, essencialmente, a de Fomentador de Saúde. Seu objetivo é a vida, a chance e a diversidade de manifestações desta, a qualidade desta vida em movimento e em interação com outras tantas expressões de vida" (São Paulo, 1992).

Para Ghirardi (1999), a proposição dos CeCCos identifica-se:

"com a proposta de reabilitação de paradigma social justamente por procurarem atribuir novos significados às ações de reabilitação, tornando-as mais complexas à medida que a elas acrescentavam outros atores sociais, modificando a cena e o espaço em que se desenrola a reabilitação" (p. 45).

\section{A convivência e as oficinas}

A concretização desta proposta seria efetivada através da convivência entre as pessoas portadoras de deficiência física, mental ou sensorial, pessoas portadoras de transtorno mental, idosos, crianças e adolescentes em situação de risco pessoal e social, denominada 'população alvo', e a população em geral. Os lugares que potencializam esse encontro entre os segmentos populacionais seriam espaços públicos, tais como parques, praças, centros esportivos ou desportivos e centros comunitários municipais, locais esses escolhidos para a implantação dos CeCCos.

A recepção daqueles que procurariam o $\mathrm{CeCCo}$, encaminhado ou não por outro serviço, se daria de forma diferenciada de outros equipamentos de saúde. Isto ocorreria pelo fato do CeCCo constituir-se essencialmente por ações abertas e dessa forma "já proporcionando o mínimo de contato interpessoal $e$ oferta de atividades que possam promover uma aproximação de vida, não burocrática, com quem o procura" (São Paulo, 1992).

O CeCCo deveria manter um sistema de referência e contra-referência com os demais equipamentos de saúde, de forma que a população alvo fosse encaminhada. No caso dos indivíduos portadores de transtorno mental seria pré-condição o acompanhamento em Unidade Básica de Saúde (São Paulo, 1992).

A convivência entre os 'diferentes' e os 'normais' deveria se dar através da realização de oficinas que desenvolveriam atividades artesanais, expressivas, esportivas, culturais, de artes plásticas, horta, jardinagem, etc. As atividades partiriam das necessidades da população usuária valorizando-se sua história e sua identidade sócio-cultural (LOPES, 1999).

Os profissionais, ao realizar as oficinas teriam como objetivo proporcionar uma reflexão sobre as 
causas do sofrimento dos indivíduos, as quais se traduzem pela falta de oportunidades e pelas limitações presentes em suas condições de vida. Assim:

se buscará no dia-a-dia da convivência, o questionamento, a reflexão e o enfrentamento conjunto evitando negação da realidade ou mesmo atitudes de compaixão, racionalização e/ou explicações religiosas/místicas, principalmente por parte daqueles que monitoram, assumem a coordenação ou acompanham voluntariamente os trabalhos, sendo de responsabilidade do corpo técnico, subsidiar e supervisionar este desenvolvimento diário e promover discussões sistematizadas (São Paulo, 1992).

\section{A equipe profissional}

As oficinas seriam mediadas por uma equipe multidisciplinar de profissionais: da área da saúde fonoaudiólogo, terapeuta ocupacional, psicólogo, assistente social, educador em saúde pública e auxiliar de enfermagem; da área da educação - educador artístico e educador físico; da cultura - agente cultural e agente artístico (os chamados oficineiros); além do pessoal de apoio (auxiliar administrativo, servente e vigia) e voluntários (São Paulo, 1992).

A atuação desta equipe multidisciplinar dar-seia através da contribuição específica de cada profissional, consorciando um novo saber.

\begin{abstract}
Os profissionais do CeCCo trabalham o indivíduo enquanto ser integral, numa relação consigo e com o mundo (sociedade, direitos trabalho, educação, comunicação relações interpessoais), tendo a expectativa que estas áreas possam contribuir para a leitura do homem, adequando instrumentais, revendo abordagens, setting (...). Cada especificidade deverá contar fundamentalmente com a sensibilidade individual de cada profissional (...) e com a disposição para a mudança (São Paulo, 1992).
\end{abstract}

Neste sentido, o técnico ao intervir na vida cotidiana do indivíduo, lidando com a sua realidade torna um tanto mais complexa a sua ação profissional, pois:

perde seu espaço de interventor privilegiado no processo de reabilitação, de detentor de um saber necessário e suficiente nas definições de tratamento e 'cura' individual. Perde o seu setting clínico tradicional e passa a agir a partir de outro local, ou seja, do lugar de alguém que auxilia o processo de inserção do indivíduo em seu ambiente, buscando contemplar as necessidades explicitadas pelo cliente e compreendidas nas condições sociais em que este está imerso. O técnico tem no cliente, então, não o seu paciente, mas um co-participante de todo o processo de reabilitação (GHIRARDI, 1999, p. 45).

Consta da proposta do CeCCos, a ocorrência periódica de reuniões internas dos membros da equipe profissional, ocasião em que seriam discutidas as ações gerais do CeCCo, seriam apontadas propostas de mudança, etc. Também deveriam acontecer as reuniões da comissão gestora, da qual participariam representantes da população usuária, de funcionários, de movimentos sociais ou de outras Secretarias Municipais e o Coordenador do CeCCo, com o objetivo de encaminhar de forma deliberativa as propostas de planejamento do CeCCo. Externamente ocorreriam: reuniões administrativas convocadas a nível central, regional ou local, com a representação de um membro da equipe; reuniões dos grupos de tarefa, da qual participaria representantes de funcionários e coordenadores de todos os CeCCos, a fim de estruturarem-se melhor as ações e trocar reflexões sobre a realidade dos $\mathrm{CeCos}$; e, por fim, as reuniões do fórum distrital e/ou regional onde seria discutida a integração de serviços e ações, o funcionamento do sistema de referência e contra-referência (São Paulo, 1992).

Além dessas reuniões, foi prevista na proposta do CeCCo a realização de cursos e seminários.

\section{A intersetorialidade}

Outra característica dessa proposta foi a intersetorialidade; outras Secretarias estariam envolvidas e trabalhariam juntas para sua concretização. A coordenação e a contratação da equipe técnica da área da saúde ficariam a cargo da Secretaria Municipal da Saúde, enquanto a contratação de outros funcionários seria responsabilidade de outras Secretarias, como no caso dos oficineiros que dependeria do repasse de verbas da Secretaria Municipal da Saúde para a Secretaria Municipal da Cultura.

Para citar, outros serviços municipais seriam:

(...) a Secretaria Municipal de Abastecimento (para auxiliar na implantação e dar assistência técnica às hortas comunitárias nos $\mathrm{CeCCos}$, bem como para estudar a viabilização da colocação da produção em equipamentos municipais de abastecimento); (...) a Secretaria Municipal de Transportes (que investiria em uma política de transporte adaptado para portadores de necessidades especiais, facilitando o acesso da população alvo dos $\mathrm{CeCCos}$ aos espaços da cidade, em linhas próprias, conectadas ao metrô) (LOPES, 1999 , p. 304-305). 


\section{Os núcleos de trabalho - as Cooperativas}

Em sua proposta, também era função do $\mathrm{CeCCo}$ a promoção de núcleos de trabalho, com o objetivo de retomar a:

participação no mundo do trabalho e numa dada produção: de bens, de serviços, de conhecimento regidos pelo princípio da convivência e da cooperação. Entendemos por princípios de convivência e cooperação fundamentalmente uma nova qualidade de relações marcando contratos afetivos e de trabalho, ou seja, a decodificação do que são consideradas limitações e deficiências - em diferenças, e esta heterogeneidade gerando produções conjuntas, concebendo assim as pessoas num novo patamar de concepção de homem e de mundo (São Paulo, 1992, grifo no original).

O que regeria esta proposição de trabalho seria a cooperação, através da colaboração do afeto, do respeito e do investimento somatório que deveria chegar numa produção conjunta. Isto é o que sustentaria a estrutura de produção, visto que cada um iria produzir de acordo com as suas capacidades de forma que o resultado desta produção, o lucro, fosse igualmente distribuído entre os usuários do CeCCo.

$\mathrm{O}$ investimento para o desenvolvimento dos núcleos de trabalho caberia ao serviço público e a comercialização resultaria na 'devolução' deste investimento na forma de materiais de consumo, sendo a diferença do montante recebido dividido igualitariamente, exercendo-se assim a divisão da remuneração pelo trabalho cooperado (São Paulo, 1992).

Não caberia ao CeCCo realizar convênios com empresas, fábricas ou indústrias, mas sim priorizar o "estímulo e a assessoria ao consórcio de usuários na construção e invenção de suas formas de produção" (SMS, 1992). A equipe multidisciplinar teria um papel importantíssimo de leitura da dinâmica das relações que se estabelecem, procurando assessorar a experiência de re-significação de autonomia e dignidade (São Paulo, 1992).

\section{O início}

Em 1989, o projeto CeCCo foi iniciado, experimentalmente, no Parque Chico Mendes, depois no Parque do Carmo, no Centro Desportivo da Freguesia do Ó e na Vila Brasilândia.

O primeiro CeCCo a ser oficialmente inaugurado foi o do Parque do Carmo, em Itaquera, em janeiro de 1990, através da primeira portaria intersecretarial SMS/ SSO-DEPAVE; o segundo CeCCo oficialmente inaugurado foi o da Freguesia do Ó em fevereiro do mesmo ano e, a partir daí, outros $16 \mathrm{CeCCos}$ foram sendo implantados durante a gestão 89/92.

Em 2000, ao final da gestão de Celso Pitta (97/ 2000), a cidade de São Paulo tinha 14 CeCCos em funcionamento, 12 deles contavam com terapeutas ocupacionais em suas equipes.

\section{DISCUSSÃO DOS RESULTADOS}

Dentre os resultados encontrados, podemos constatar que os CeCCos lograram se constituir em importantes inovações de intervenção na área social, com interfaces no campo da saúde, da cultura e da educação, apesar das dificuldades para a sua manutenção nas gestões seguintes a de sua criação. Os CeCCos, ao proporem um serviço novo e diferenciado, apresentavam fragilidades já em sua implantação. Devido ao desconhecimento natural daquilo que é novo e que implica novos mecanismos e ações, os impasses da proposta mereceriam maiores atenções para que pudessem ser trabalhados e sanados, como a questão do desenvolvimento das cooperativas. Pelo que percebemos isto não ocorreu. Ao contrário, após a implantação do PAS, quatro dos dezoito $\mathrm{CeCCos}$ existentes e em fase de implantação até então, foram desativados. Dos quatorze CeCCos em funcionamento na cidade de São Paulo*, alguns equipamentos, no mínimo três deles, adquiriram um caráter institucionalizador, conforme Basagalia F. e Basaglia F.O. (1979) e Goffman (1974), em função, sobretudo da localização em espaços fechados ao público em geral, o que fugia totalmente à concepção e à proposição deste serviço. Por outro lado, pode-se considerar que a maioria dos CeCCos busca desenvolver suas ações em direção ao que foi definido em sua Normatização**.

A questão da formação das cooperativas nos CeCCos, nestes dez anos de sua existência, é algo que ainda demanda maiores discussões e trabalhos, pois muito pouco foi implementado. Consideramos que um dos prováveis motivos para o não desenvolvimento dessas cooperativas é que somente nos últimos anos,

\footnotetext{
Dados obtidos até dezembro de 2000 .

** A Normatização (São Paulo, 1992) contém as proposições dos Centros de Convivência e Cooperativas e, elaborada ao final de 1992, não foi de fato aprovada pela Legislação Municipal. Permaneceu, entretanto, como diretriz para técnicos e coordenadores).
} 
especialmente no final da década de 90 , as formas de trabalho cooperado e a economia solidária, têm sido mais amplamente problematizadas e debatidas.

Constatamos, por outro lado, que inúmeros são os benefícios proporcionados por estes equipamentos, dos quais se destacam: o estabelecimento da convivência entre as populações, que é um dos objetivos da proposta na direção da luta contra a discriminação e a segregação social; o aumento da qualidade de vida, minimizando muitas vezes processos de cronificação de doenças; e o papel de fomentador de cidadania.

\section{atividade \\ O CeCCo, o terapeuta ocupacional e a}

Procuramos compreender na pesquisa realizada, através de entrevistas e, sobretudo, da observação da prática desenvolvida, qual o papel, a função do terapeuta ocupacional; como a sua atuação influencia a equipe; e em que medida o uso do recurso atividade o diferencia dos outros profissionais que atuam no CeCCo com o mesmo recurso. Alguns pontos que demarcam essas diferenças são apontados a seguir.

De uma maneira geral pode-se dizer que a contribuição do terapeuta ocupacional no CeCCo gira em torno da maneira como 'olha', compreende e intervêm numa dada situação.

Para os demais técnicos entrevistados, os terapeutas ocupacionais ao chegarem nos CeCCos, são os profissionais da equipe que demonstram mais tranqüilidade e disponibilidade para entender a sua proposta e enfrentar algumas questões como, por exemplo, o fato do trabalho nas oficinas ser muito aberto e amplo, em comparação ao trabalho desenvolvido em um ambulatório, ou em uma UBS, ou ainda em um hospital-dia ou CAPS (Centros de Atenção Psico-social), com um enfoque mais clínico. A formação acadêmica, nesse sentido, foi avaliada como uma base importante para a atuação no serviço, sendo que o aprendizado em torno da inclusão/inserção social, da dinâmica de grupos, bem como da facilitação do convívio do indivíduo em seu meio e no seu cotidiano e de toda a gama de atividades aprendidas, vem ao encontro do trabalho desenvolvido no CeCCo.

Quanto à utilização da atividade, observamos que o terapeuta ocupacional frente à atividade postase com menos pré-conceitos do que outros profissionais, não atribuindo um juízo de valor a partir de seus julgamentos sociais ou emocionais para esta ou aquela atividade. Não delegando sentidos para a atividade, busca, antes, o sentido e o significado atribuídos pelo próprio indivíduo ou pelo grupo. Demonstra ter um cuidado diferenciado com o material e o produto final das atividades dos usuários. Neste sentido, o terapeuta ocupacional procura manter-se aberto ao processo do outro, por conta mesmo de sua formação, e desse modo, em relação aos outros profissionais, carrega em suas intervenções, uma noção singular quanto ao uso das atividades.

O terapeuta ocupacional também se diferencia de outros técnicos ao utilizar a atividade, analisando e adaptando-a, no momento de sua execução, bem como busca lidar com o indivíduo a partir de suas demandas, a fim de atender às suas necessidades, observando a dinâmica das suas relações com as outras pessoas, com o seu trabalho e com a sua atividade.

Além disso, notamos que o enfoque na ação é outro diferencial da visão do terapeuta ocupacional. Assim, uma das primeiras preocupações deste profissional refere-se a uma visão do 'fazer' e de tudo o que norteia esse processo; o produto final é trabalhado em um outro momento ou em um segundo plano. Essa forma de lidar com a atividade, o terapeuta ocupacional transmite claramente em suas intervenções no CeCCo e proporciona um aprendizado, principalmente, aos oficineiros e voluntários, que, ao chegarem no serviço procuram ensinar a técnica e focalizam o produto final da oficina. Isso poderia dar ao CeCCo e às suas oficinas uma característica de 'aula' ou 'curso', de estar ali para aprender uma técnica prioritariamente e não se buscando a convivência nos processos grupais. Os oficineiros foram reformulando e adquirindo uma outra postura diante do usuário, principalmente, da população alvo, passando a compreender a proposta do serviço.

Observamos ainda, como contribuição do terapeuta ocupacional no que se refere ao trabalho em equipe, uma reflexão acerca do funcionamento e dos conteúdos das oficinas de acordo com as necessidades dos usuários. Isto foi bastante apontado pelos demais profissionais*.

Por outro lado, podemos afirmar, com base em entrevistas de técnicos e coordenadores dos CeCCos que não contam com terapeutas ocupacionais**, mas onde seu trabalho é conhecido, que sua ausência, como a de qualquer outro técnico que compõe a equipe de um CeCCo, vai implicar perdas em termos qualitativos,

\footnotetext{
* Os outros profissionais entrevistados são, em sua maioria, psicólogos, e, também, assistentes sociais, educadores em saúde pública e educadores físicos. ${ }^{* * *}$ Durante a pesquisa encontramos dois CeCCos nos quais estavam ausentes os terapeutas ocupacionais, mas que tiveram em seu quadro este profissional. Outros dois CeCCos não tinham temporariamente o terapeuta ocupacional por dificuldades em preencher a vaga
} 
tanto no que se refere à sua contribuição na equipe multidisciplinar quanto na intervenção junto ao usuário em oficina.

O terapeuta ocupacional com sua especificidade, que inclui, mas não se restringe ao uso do recurso "atividades", tem contribuído de forma importante para o desenvolvimento das ações dos CeCCos.

Os CeCCos, ao realizarem seus propósitos, vão além do que a sociedade ainda concebe como 'saúde'. Por ser uma prática inovadora, por um lado, não encontrou o espaço e o reconhecimento por parte das administrações municipais (gestões 93/96 e 97/2000) que legaram um enorme sucateamento na área da saúde, o não investimento nos recursos humanos e materiais e a imposição do PAS.

Por outro lado, constatamos que os $\mathrm{CeCCos}$ representam uma inovação sócio-política (OFFE; LENHARDT, 1984) que permaneceu, dado, principalmente, o conhecimento adquirido por parte da população usuária que passou a reivindicar esse serviço.

Dessa forma, podemos concluir quanto aos CeCCos que produzir saúde no seu sentido mais amplo e propiciar o exercício de direitos de cidadania aos indivíduos não é uma utopia, quando temos ferramentas como esses equipamentos.

A partir da experiência dos $\mathrm{CeCCos}^{*}$ nos quais foi possível uma maior participação, notou-se que a terapia ocupacional possui um espaço importante neste equipamento pelo fato de que os fundamentos da profissão vêm ao encontro dessa proposta de produzir e conceber saúde e, principalmente, pelo uso do recurso "atividade" para a busca da autonomia e da participação social.

A especificidade do terapeuta ocupacional, está muito mais na visão/compreensão que adquire através da formação e de sua prática e refere-se à forma como apreende o indivíduo dentro de suas relações e no seu fazer cotidiano.

Constatamos, finalmente, que os terapeutas ocupacionais, esses novos técnicos inseridos no serviço público municipal, a partir de 1989, encontraram seus espaços e contribuíram, assim como os outros profissionais, para a implantação e manutenção dos CeCCos, sobretudo com a utilização dos recursos "atividades", "oficinas" e "grupos", os quais apresentamse como importantes eixos para o acolhimento e para a inclusão social da população usuária.

LOPES, R. E.; LEÃO, A. Occupational therapists and "Centro de Convivencia e Cooperativas": new health actions. Rev. Ter. Ocup. Univ. São Paulo, v. 13, n. 2, p. 56-63, maio/ago. 2002.

\begin{abstract}
In São Paulo city, Brazil, the public administration 1989-1992 suggested specific policies for the development of health actions directed to persons with physical and mental problems. This population was previously excluded from the public health services and needed to be assisted, in accordance to the 'Sistema Único de Saúde', 'SUS' (which can be freely translated as 'Health System for all citizens'). New health services were established, like 'CeCCos' (Community Centers), and jobs were created for occupational therapists, in order to supply the services required by the population. In this work, we studied the changes in São Paulo's health politics, during the period 1989-2000, analyzing their influence on the CeCCos's abilities to fulfill their purposes. We discussed the contribution of the occupational therapists to the CeCCos; we also sought to understand the ways in which this professional uses the resource 'activities', as compared to other health staff. We found conclusive evidence of the innovative potential of the CeCCos; the occupational therapists - and their use of the resource 'activities' as a tool for social insertion - certainly had a relevant role in their development.
\end{abstract}

KEYWORDS: Occupational therapy/manpower. Cooperative behavior. Delivery of health care.

Patient care team/trends. Health police/trends.

\footnotetext{
* Sobretudo do CeCCo Parque São Domingos que mantém em sua equipe desde a sua implantação uma terapeuta ocupacional como coordenadora da unidade.
} 


\section{REFERÊNCIAS}

BARROS, D. D.; LOPES, R. E.; OLIVER, F. C. Novas propostas assistenciais em São Paulo: estudo da incorporação da terapia ocupacional no contexto das ações de saúde mental e saúde da pessoa portadora de deficiência física, sensorial e/ou mental, no município de São Paulo. São Paulo: USP/UFSCar/CNPQ, 1995. 89p. (Relatório de pesquisa)

BASAGLIA, F., BASAGLIA, F. O. O homem no pelourinho. São Paulo: Tradução IPSO - Instituto de Psiquiatria Social, 1979.

BRASIL. Constituição: República Federativa do Brasil. Brasília: Centro Gráfico, 1988.

CONH, A.; ELIAS, P. E. (Coord.). O processo de implantação do PAS: universalidade ou seletividade no acesso à saúde? São Paulo: Cortez/CEDEC, 1997. (Relatório de pesquisa)

GHIRARDI, M. I. G. Representações da deficiência e práticas de reabilitação: uma análise do discurso técnico. 1999. 151p. Tese (Doutorado). Instituto de Psicologia, Universidade de São Paulo, São Paulo, 1999.

GOFFMAN, E. Prisões, manicômios e conventos. São Paulo: Perspectiva, 1974.

LOPES, R. E. Cidadania, políticas públicas e terapia ocupacional, no contexto das ações de saúde mental e saúde da pessoa portadora de deficiência, no município de São Paulo. Campinas, 1999. 539p. Tese (Doutorado em Educação) - Faculdade de Educação da UNICAMP, CAMPINAS, 1999.

OFFE, C.; LENHARDT, G. Teoria do estado e política social. In: PROBLEMAS estruturais do estado capitalista. Rio de Janeiro: Tempo Brasileiro, 1984. p. 9-53.

SÃo PAUlO (Município). Secretaria de Saúde. Normatização das ações desenvolvidas nos serviços de saúde mental. São Paulo, 1992.

Recebido para publicação: 14/03/2002

Aceito para publicação: 23/04/2002 